\title{
Nonalcoholic fatty liver disease and risk of incident chronic kidney
}

\section{disease: an updated meta-analysis}

Alessandro Mantovani, $\mathrm{MD}^{1}$, Graziana Petracca, $\mathrm{MD}^{1}$, Giorgia Beatrice, $\mathrm{MD}^{1}$, Alessandro

Csermely, $M D^{1}$, Amedeo Lonardo, $M D^{2}$, Jörn M. Schattenberg, $\mathrm{MD}^{3,4}$, Herbert Tilg, $\mathrm{MD}^{5}$,

Christopher D. Byrne, MB BCh, $\mathrm{PhD}^{6,7}$, Giovanni Targher, $\mathrm{MD}^{1}$

${ }^{1}$ Section of Endocrinology, Diabetes and Metabolism, Department of Medicine, University and Azienda Ospedaliera Universitaria Integrata of Verona, Verona, Italy

${ }^{2}$ Department of General Internal Medicine, of Urgence and Post-Acute, Ospedale Civile di Baggiovara, Modena, Italy

${ }^{3}$ Department of Medicine 1, University Medical Center Mainz, Mainz, Rheinland-Pfalz, Germany

${ }^{4}$ Metabolic Liver Research Program, University Medical Center Mainz, Mainz, Rheinland-Pfalz, Germany

${ }^{5}$ Department of Internal Medicine I, Gastroenterology, Hepatology, Endocrinology \& Metabolism, Medical University Innsbruck, Innsbruck, Austria

${ }^{6}$ Nutrition and Metabolism, Faculty of Medicine, University of Southampton, UK

${ }^{7}$ Southampton National Institute for Health Research Biomedical Research Centre, University Hospital Southampton, Southampton General Hospital, Tremona Road, Southampton, UK

Running title: NAFLD and increased CKD incidence

Word count: abstract 248; text 3,934 (excluding title page, abstract, references, figure legends and tables); n. 1 Table + n. 2 Figures + online-only supplementary Material ( $\mathrm{n} .4$ supplementary Tables + n. 3 supplementary Figures).

\section{Address for correspondence:}

Prof. Giovanni Targher, MD

Section of Endocrinology, Diabetes and Metabolism

Department of Medicine

University and Azienda Ospedaliera Universitaria Integrata

Piazzale A. Stefani, 1

37126 Verona, Italy

Phone: +39-045-8123110

E-mail: giovanni.targher@univr.it 


\section{ABSTRACT}

Objective: Studies reported a significant association between nonalcoholic fatty liver disease (NAFLD) and increased risk of chronic kidney disease (CKD). However, whether this risk changes with increasing severity of NAFLD remains uncertain. We performed a meta-analysis of observational studies to quantify the magnitude of the association between NAFLD and incident CKD.

Design: We systematically searched PubMed, Web of Science and Scopus from January 2000 to August 2020 using predefined keywords to identify observational studies with a follow-up duration of $\geq 1$ year, in which NAFLD was diagnosed by blood biomarkers/scores, International Classification of Diseases codes, imaging techniques or liver biopsy. Data from selected studies were extracted, and meta-analysis was performed using random-effects modelling.

Results: 13 studies with 1,222,032 individuals ( $28.1 \%$ with NAFLD) and 33,840 cases of incident CKD stage $\geq 3$ (defined as estimated glomerular filtration rate $<60 \mathrm{ml} / \mathrm{min} / 1.73 \mathrm{~m}^{2}$, with or without accompanying overt proteinuria) over a median follow-up of 9.7 years were included. NAFLD was associated with a moderately increased risk of incident CKD ( $n=10$ studies; random-effects hazard ratio $\left.[\mathrm{HR}] 1.43,95 \% \mathrm{Cl} 1.33-1.54 ; I^{2}=60.7 \%\right)$. All risks were independent of age, sex, obesity, hypertension, diabetes and other conventional CKD risk factors. Sensitivity analyses did not alter these findings. Funnel plot did not reveal any significant publication bias.

Conclusion: This large and updated meta-analysis indicates that NAFLD is significantly associated with a $\sim 1.45$-fold increased long-term risk of incident CKD stage $\geq 3$. Further studies are needed to examine the association between the severity of NAFLD and long-term risk of incident CKD.

Keywords: NAFLD; fatty liver; CKD risk; meta-analysis 


\section{SUMMARY BOX}

What is already known about this subject: Observational studies have shown that nonalcoholic fatty liver disease (NAFLD) is associated with an increased incidence of chronic kidney disease (CKD stage $\geq 3$ ), but currently it is uncertain whether this risk changes with the severity of NAFLD.

What are the new findings: This meta-analysis of 13 observational longitudinal studies involving nearly 1,200,000 middle-aged individuals ( $28.1 \%$ with NAFLD; $n=343,248$ ) from different countries indicates that the long-term risk of developing CKD stage $\geq 3$ is increased 1.45 fold in individuals with NAFLD. This risk seems to parallel the severity of NAFLD, especially the severity of liver fibrosis. However, more studies are required to further support this finding.

How might it impact on clinical practice in the foreseeable future: Health care professionals should be aware that risk of incident CKD stage $\geq 3$ is moderately increased in patients with NAFLD. These findings call for a more active and systematic search for CKD among patients with NAFLD with a view to potential earlier treatment and referral to a nephrologist. 


\section{INTRODUCTION}

Nonalcoholic fatty liver disease (NAFLD) is the predominant cause of chronic liver diseases worldwide, causing considerable liver-related and extra-hepatic morbidity and mortality. Indeed, NAFLD affects up to $\sim 30 \%$ of the general adult population both in the Europe and in the United States and its global prevalence is projected to further increase in the foreseeable future [1-3].

Accumulating evidence indicates that NAFLD is associated with an increased prevalence and incidence of chronic kidney disease (i.e. a condition defined by a glomerular filtration rate of less than $60 \mathrm{ml} / \mathrm{min} / 1.73 \mathrm{~m}^{2}$, namely CKD stage $\geq 3$ ) [4,5], which is an established risk factor for end-stage kidney disease, cardiovascular disease and premature mortality [6]. Evidence also supports the notion that NAFLD and CKD share many cardio-metabolic risk factors, as well as pro-inflammatory and pro-fibrotic molecular pathways [5].

To date, there are only two previous meta-analyses (published in 2014 and 2018, respectively) that have examined the association between NAFLD and risk of incident CKD $[7,8]$. However, both meta-analyses had a relatively small sample size and included only a few non-Asian cohort studies. Notably, over the last two years a number of large cohort studies in adults from the United States and Europe have examined the association between NAFLD and longterm risk of incident CKD stage $\geq 3$. In addition, both of these meta-analyses did not definitively address the question of whether the strength of any association (between NAFLD and risk of incident CKD) was affected by increasing severity of NAFLD.

We have therefore undertaken an updated meta-analysis of observational studies of adults from different countries examining the association between NAFLD and risk of incident CKD stage $\geq 3$. Clarification of the magnitude of risk of incident CKD associated with the different stages of liver disease within the spectrum of NAFLD might have direct relevance for primary preventive strategies against CKD. Thus, the specific aims of this meta-analysis were to precisely gauge the nature and magnitude of the association between NAFLD and risk of 
incident CKD stage $\geq 3$; and to assess whether the severity of NAFLD was associated with an increased risk of incident CKD stage $\geq 3$.

\section{METHODS}

Registration of review protocol

The protocol for this systematic review was registered in advance with Open Science Framework registries (no: osf.io/4d8vf).

\section{Data sources and searches}

We systematically searched PubMed, Scopus and Web of Science databases from January 1, 2000 to August 15, 2020 (date last searched) to identify observational studies assessing the association between NAFLD and risk of incident CKD stage $\geq 3$. Search free text terms were "nonalcoholic fatty liver disease" ("NAFLD" OR "fatty liver" OR "nonalcoholic steatohepatitis") AND "chronic kidney disease" OR "risk of chronic kidney disease" OR "incident CKD" OR "incidence of CKD". Searches were limited to human studies without any language restriction. Reference lists of relevant papers and review articles were hand searched for other relevant studies not covered by the original database searches. We performed a systematic review in accordance with the Preferred Reporting Items for Systematic Reviews and Meta-Analyses (http://www.prisma-statement.org). Because the included studies were observational in design, we followed the reporting proposed by the Meta-analysis Of Observational Studies in Epidemiology (MOOSE) for the meta-analysis of these studies [9].

\section{Study selection}

The criteria for inclusion of the meta-analysis were as follows: 1) studies with a follow-up duration of $\geq 1$ year that explored the association between NAFLD and risk of incident CKD; 2) studies reporting hazard ratios (HRs) or odds ratios (ORs) with 95\% confidence intervals (95\% Cls) values for the outcome of interest; 3) studies where the diagnosis of NAFLD was based on serum liver enzymes (mostly gamma-glutamyltransferase [GGT] levels), blood biomarkers/scores (e.g. fatty liver index or other algorithmically-derived scores to diagnose 
the presence of hepatic steatosis), International Classification of Diseases (ICD) codes, imaging techniques (mostly ultrasonography) or biopsy in the absence of significant alcohol consumption (i.e. usually defined in the eligible studies as either alcohol intake $>20 \mathrm{~g} /$ day for both sexes, or $>30 \mathrm{~g} /$ day for men and $>20 \mathrm{~g} /$ day for women, respectively) and other competing causes of hepatic steatosis; and 4) studies where the diagnosis of CKD was based on the occurrence of eGFR $<60 \mathrm{ml} / \mathrm{min} / 1.73 \mathrm{~m}^{2}$ [as estimated by either the four-variable Modification of Diet in Renal Disease (MDRD) or the Chronic Kidney Disease Epidemiology Collaboration (CKD-EPI) study equations], with or without accompanying overt proteinuria, during follow-up. Study participants included in the meta-analysis were of either sex without any restriction of age, race, ethnicity or co-morbidities.

Criteria for exclusion were as follows: 1) congress abstracts, case reports, theses, reviews, practice guidelines, editorials, and cross-sectional studies; 2) studies with a follow-up duration $<1$ year; 3) studies which did not exclude individuals with significant alcohol consumption and other competing causes for hepatic steatosis; 4) studies which did not specifically report any HRs and $95 \% \mathrm{Cls}$ for the outcome of interest; 5) studies where the outcome was defined as occurrence of early CKD stages (CKD stages 1 and 2; i.e. abnormal albuminuria or overt proteinuria with an eGFR value $\geq 60 \mathrm{ml} / \mathrm{min} / 1.73 \mathrm{~m}^{2}$ ); and 6) studies conducted in the paediatric population ( $<18$ years) or in people with type 1 diabetes.

\section{Data extraction and quality assessment}

Two investigators (AM and GP) independently examined all titles and abstracts, and obtained full texts of potentially relevant papers. Working independently and in duplicate, we read the papers and determined whether they met inclusion criteria. Discrepancies were resolved by consensus, referring back to the original article, in consultation with a third author (GT).

For all studies, we extracted information on study design, study country, population characteristics, methods used for NAFLD diagnosis, length of follow-up, outcome of interest, matching and confounding factors included in multivariable regression analyses. In case of multiple publications, the most up-to-date or comprehensive information was included. 
Two authors assessed the risk of bias independently (AM and GP). Any discrepancies were addressed by a re-evaluation of the original article by a third author (GT). We also contacted two corresponding authors of the eligible studies in order to obtain additional information for the meta-analysis, but only one responded providing extra-information to us (as reported in the Acknowledgments section). Since all the included studies were non-randomised and had a cohort design, the Newcastle-Ottawa Scale (NOS) was used to judge study quality, as recommended by the Cochrane Collaboration [10]. This scale uses a star system (with a maximum of 9 stars) to evaluate a study in three domains: selection of participants, comparability of study groups, and ascertainment of outcomes of interest. We judged studies that received a score of 9 stars to be at low risk of bias, studies that scored 7 or 8 stars to be at medium risk, and those that scored $\leq 6$ stars to be at high risk of bias [10].

\section{Data synthesis and analysis}

The primary outcome was the development of incident CKD stage $\geq 3$ (as defined above) among individuals with NAFLD compared to NAFLD-free controls. The HRs (or ORs) and 95\% Cls were considered as the effect size for each eligible study. In the case of studies reporting HRs with varying degrees of covariate adjustment, those that reflected the maximum extent of adjustment for potentially confounding factors were extracted. The adjusted HR/ORs of all eligible studies were then pooled, and an overall estimate of effect size was calculated using a random-effects model, as this methodology considers any differences between studies even if there is no statistically significant heterogeneity [10].

Visual inspection of the forest plots was used to evaluate statistical heterogeneity. This was also assessed with the $I^{2}$-statistics, which provides an estimate of the percentage of variability across eligible studies that is due to heterogeneity rather than chance alone. A rough guide to interpretation is as follows: $I^{2}$-values of approximately $25 \%$ represent low heterogeneity; approximately 50\% represent medium heterogeneity; and approximately $75 \%$ represent high heterogeneity [11]. The risk of publication bias was evaluated using the funnel plot and the rank correlation Begg's test $[10,12]$. 
To explore the possible sources of heterogeneity among the eligible studies and to test the robustness of the associations, we conducted stratification-sensitivity analyses by study country, length of study follow-up, methodology used for the diagnosis of NAFLD, severity of NAFLD (based on either the coexistence of hepatic steatosis on ultrasonography and elevated serum GGT levels or the severity of liver fibrosis by histology and/or non-invasive fibrosis scores, such as Fibrosis [FIB]-4 index or NAFLD fibrosis score [NFS])[13,14], whether the studies had 8 or 9 stars on the NOS scale (i.e. the "high-quality" studies), or whether the studies had full adjustment for traditional CKD risk factors [i.e. arbitrarily defined as those studies adjusting for at least age, sex, body mass index, smoking, pre-existing diabetes (or plasma glucose levels), hypertension, dyslipidemia (or plasma lipid levels) and baseline eGFR]. We also performed meta-regression analyses for the association of age, sex, body mass index, pre-existing diabetes and hypertension with NAFLD-related HRs of incident CKD. Finally, we tested for possible excessive influence of individual studies using a meta-analysis influence test that eliminated each of the included studies one at a time.

All statistical tests were two sided and used a significance level of $p<0.01$ (i.e. $p=0.05 / 5$ comparisons, since we performed an overall primary analysis +4 stratification-sensitivity analyses, as specified above). We used STATA ${ }^{\circledR} 14.0$ (StataCorp, College Station, Texas, USA) for all statistical analyses. In supplementary Table $\mathbf{S 1}$ are reported the STATA ${ }^{\circledR}$ codes used for the meta-analysis.

\section{RESULTS}

\section{Characteristics of included studies}

Figure 1 summarizes the results of the literature research and study selection. Based on the titles and abstracts of 662 citations, we initially identified 20 potentially eligible studies [1534] from three electronic databases prior to August 15, 2020. After examining the full text of these publications, we excluded 7 studies [15,19,30-34], due to unsatisfactory inclusion 
criteria or unsatisfactory outcome measures as specified in the PRISMA flow diagram. Therefore, 13 studies were eligible for inclusion in the meta-analysis and were assessed for quality. In supplementary Table $\mathbf{2}$ are specified the syntax used and the records identified through database searching.

The main characteristics of the included studies are summarized in supplementary Table S3. All the eligible studies had an observational (retrospective or prospective) design. Most of these studies recruited participants either from general populations or from outpatient cohorts of individuals with diabetes, in which NAFLD was diagnosed by serum liver enzymes, blood biomarkers/scores, ICD-9/ICD-10 codes, imaging techniques or liver biopsy.

Overall, in the 13 studies included in the meta-analysis there were 1,222,032 middle-aged individuals ( $53.6 \%$ men; mean age 51.7 years; mean BMI $25.7 \mathrm{~kg} / \mathrm{m}^{2}$ ) with a total of 343,248 (28.1\%) individuals with NAFLD at baseline and 33,840 cases of incident CKD stage $\geq 3$ over a median follow-up of 9.7 years (inter-quartile range: 5-10 years). Seven studies were carried out in Asia (South Korea, Japan and China); four studies were carried out in Europe (Sweden, Finland, Germany and Italy) and two studies were carried out in the United States. Four studies used ultrasonography for the diagnosis of $\operatorname{NAFLD~(~} n=57,130$ individuals included), one study used multi-detector computed tomography ( $n=688$ ), four studies used serum liver enzyme levels or blood biomarkers/scores $(n=35,054)$, two studies used ICD-9/ICD-10 codes ( $n=1,128,611)$, and two studies used liver histology ( $n=599)$. In all studies, the subjects had an eGFR value $\geq 60 \mathrm{ml} / \mathrm{min} / 1.73 \mathrm{~m}^{2}$ without overt proteinuria at baseline, whereas incident CKD during the follow-up was defined as at least stage 3 CKD (i.e. eGFR $<60 \mathrm{ml} / \mathrm{min} / 1.73 \mathrm{~m}^{2}$ estimated by either the MDRD or the CKD-EPI study equations), with or without accompanying overt proteinuria. As reported in supplementary Table S4, six studies received at least 8 stars on the NOS (i.e. studies at relatively low risk of bias), four studies received 7 stars (studies at medium risk of bias) and three studies received 6 stars (studies at high risk of bias), thus indicating an overall medium-low risk of bias. 
The distribution of studies by estimate of the association between NAFLD and risk of incident CKD is plotted in Figure 2. Ten studies [involving a total of 1,215,872 middle-aged individuals with $337,088(27.7 \%)$ patients with NAFLD and 33,533 cases of incident CKD] provided data suitable for the pooled primary analysis. We excluded three studies from this pooled primary analysis $[18,26,27]$, because they did not include NAFLD-free controls; these three studies were used for examining the association between the severity of NAFLD and risk of CKD (see below).

The presence of NAFLD was associated with a moderately increased risk of incident CKD stage $\geq 3$ ( $n=10$ studies; random-effects HR 1.43, 95\%Cl 1.33-1.54; test for overall effect Z=10.0, $\left.\mathrm{p}<0.0001 ; I^{2}=60.7 \%\right)$. Notably, since we have always used the fully adjusted HR estimates for each eligible study (as specified in supplementary Table 3 ), this pooled random-effects HR was independent of a relatively large number of conventional CKD risk factors, such as age, sex, body mass index, smoking, hypertension, dyslipidemia, diabetes, baseline eGFR, and medication use.

As also shown in Figure 2, when the comparison was stratified by methodologies used for the diagnosis of NAFLD, the association between NAFLD and the risk of CKD was consistent across studies using blood biomarkers/scores ( $n=2$ studies; random-effects HR 1.34, 95\% CI 1.20-1.49; test for overall effect $Z=5.43, p<0.0001 ; I^{2}=0 \%$ ), imaging techniques ( $n=4$ studies; randomeffects HR 1.37, 95\% CI 1.17-1.62; test for overall effect $Z=3.81, p<0.0001 ; I^{2}=27.3 \%$ ) or ICD9/ICD-10 codes ( $\mathrm{n}=2$ studies; random-effects HR 1.58, 95\%Cl 1.53-1.63; test for overall effect $\left.\mathrm{Z}=27.8, \mathrm{p}<0.0001 ; I^{2}=0 \%\right)$.

\section{Subgroup analyses and meta-regressions}

To explore possible sources of heterogeneity across the eligible studies, we carried out some subgroup/sensitivity analyses (Table 1). Notably, the significant association between NAFLD and CKD risk was consistent in all subgroups considered. In particular, the random-effects HRs were essentially superimposable when the comparison was stratified by study country, follow-up duration, NOS quality scale, or degree of covariate adjustment. 
In addition, as reported in supplementary Figure S1, the results of univariable metaregression analyses to examine the effect of potential moderator variables showed a significant positive association between the proportion of patients with hypertension at baseline and risk of NAFLD-related CKD (Figure S1, panel D). In contrast, meta-regression analyses did not reveal any significant effects of age, sex, body mass index or pre-existing diabetes on the association between NAFLD and risk of NAFLD-related CKD.

We also tested for the possibility of excessive influence of individual studies using an influence test that eliminated each of the included studies one at a time. Interestingly, eliminating each of the eligible studies from the analysis had no significant effect on the overall risk of CKD (supplementary Figure S2).

As shown in supplementary Figure S3, the rank correlation Begg's test did not show any statistically significant asymmetry of the funnel plot $(p=0.392)$, thus suggesting that publication bias was unlikely.

\section{Severity of NAFLD and risk of CKD}

Four studies [involving a total of 47,590 individuals with 20,383 (42.8\%) patients with NAFLD at baseline and 998 cases of incident CKD] reported data on patients with more 'severe' NAFLD, defined either by presence of NAFLD on ultrasonography plus elevated serum GGT concentrations [18], or by severity of liver fibrosis assessed by non-invasive fibrosis scores [23] or liver histology $[26,27]$. It is important to point out that all these studies did not have a comparator control group without NAFLD, which was the case for all the studies shown in Figure 2. We believe that the results for incident CKD stage $\geq 3$ with severity of NAFLD can only be discussed as a systematic review, not a meta-analysis, due to insufficient data suitable for combining. That said, the risk for incident CKD stage $\geq 3$ appeared to be greater amongst those with advanced fibrosis assessed by non-invasive fibrosis scores ( $n=1$ study; adjusted HR 1.59 (95\% Cl 1.31-1.93 in patients with high NFS vs. low NFS) [23] or liver histology ( $\mathrm{n}=2$ studies 
reporting HRs for incident CKD ranging from 2.75 to 3.25 in NAFLD patients with $\geq F 3$ vs. FO-2) $[27,28]$.

\section{DISCUSSION}

In this large and updated meta-analysis of 13 observational studies [involving a total of $1,222,032$ middle-aged individuals ( $28.1 \%$ with NAFLD) from different countries], we found that the presence of NAFLD conferred a hazard risk of $\sim 1.45$ for incident CKD stage $\geq 3$ (defined as defined as occurrence of eGFR $<60 \mathrm{ml} / \mathrm{min} / 1.73 \mathrm{~m}^{2}$, with or without accompanying overt proteinuria) over a median follow-up of 9.7 years (random-effects HR $1.43,95 \% \mathrm{Cl} 1.33-1.54$; test for overall effect $\left.Z=10.0, p<0.0001 ; l^{2}=60.7 \%\right)$. The magnitude of this risk remained essentially unchanged after stratification by study design, follow-up duration, covariate adjustment, NOS quality scale or modality of NAFLD diagnosis. Furthermore, the risk of incident CKD stage $\geq 3$ remained statistically significant in those studies where analysis was adjusted for age, sex, adiposity measures, smoking status, preexisting diabetes, hypertension, dyslipidemia and baseline eGFR (as shown in Table 1). Although there are insufficient data suitable for combining them into a formal meta-analysis, the risk of CKD stage $\geq 3$ seemed to be higher among NAFLD patients with greater severity of liver fibrosis (assessed by histology and/or fibrosis scores). However, additional larger studies are certainly needed to better examine this issue.

The findings of our updated meta-analysis confirm and extend the results of two previous smaller meta-analyses $[7,8]$. In the first meta-analysis that incorporated 11 longitudinal studies (involving a total of 28,680 individuals), Musso et al. [7] reported that NAFLD was associated with a nearly $80 \%$ increased risk of incident CKD (random-effects HR $1.79,95 \% \mathrm{Cl}$ 1.65-1.95). However, at variance with our meta-analysis, these authors also incorporated studies that examined the incidence of early CKD stages (i.e. abnormal albuminuria or proteinuria with eGFR $\geq 60 \mathrm{ml} / \mathrm{min} / 1.73 \mathrm{~m}^{2}$ ) [7]. In the second meta-analysis that incorporated 9 longitudinal studies with a follow-up duration of at least 1 year (published up to August 2017), involving a total of 96,595 individuals of predominantly Asian descent, our 
group reported that NAFLD was associated with a nearly $40 \%$ increased risk of incident CKD stage $\geq 3$ (random-effects HR 1.37, 95\% Cl 1.20-1.53; $I^{2}=34 \%$ )[8]. Patients with more 'severe' NAFLD (according to ultrasonographic steatosis scores or non-invasive markers of liver fibrosis) were also more likely to develop incident CKD ( $n=2$ studies; random-effects HR 1.50, $95 \% \mathrm{Cl} 1.25-1.74)$. However, no studies with biopsy-proven NAFLD were available for the analysis [8].

Compared to the results of this latter meta-analysis [8], we have increased the number of eligible studies (by including 5 large cohort studies with long follow-up durations published from August 2017 to August 2020); and we have increased the sample size more than 12 times, increasing the total number of individuals from almost 96,500 to approximately $1,200,000$. The issue of whether the increase in NAFLD-related risk of incident CKD stage $\geq 3$ is confined only to individuals with advanced NAFLD or applies to all people with NAFLD, is particularly relevant given the disease burden imposed by NAFLD worldwide. Although further cohorts of well-characterized patients with NAFLD are needed to better elucidate the association between the severity of NAFLD and the risk of incident CKD, the results of our study by including new cohort studies (that compared the risk of CKD in patients with advanced fibrosis vs. absent or moderate fibrosis assessed by histology or non-invasive fibrosis scores) suggested that the risk of incident CKD stage $\geq 3$ was higher amongst NAFLD patients with greater severity of liver fibrosis. If confirmed by future studies, these latter results are in line with previously published data showing an association between the severity of liver fibrosis and the risk of developing liver-related complications, cardiovascular disease and other extra-hepatic diseases [3,35-39].

An in-depth analysis of the putative pathophysiological mechanisms by which NAFLD may contribute to the development and progression of CKD is beyond the scope of this metaanalysis. However, to date, there is convincing evidence of biological plausibility that NAFLD increases the risk of developing CKD. Although further research is needed (e.g. Mendelian randomization studies), accumulating evidence suggests that NAFLD (especially in its more advanced forms) exacerbates hepatic insulin resistance, promotes atherogenic dyslipidemia, 
induces hypertension and causes the release of multiple proinflammatory cytokines and hepatokines that may contribute to the development and progression of CKD [5,40-43].

Our meta-analysis has some limitations that are strictly inherent to the design of the included studies. First, the observational design of the eligible studies does not allow for proving causality. Second, as shown in Supplementary Table 3, although the large majority of the eligible studies adjusted the results for age, sex, obesity, hypertension, diabetes and other conventional CKD risk factors, the possibility of residual confounding by some unmeasured factors cannot be ruled out. Indeed, some studies reported incomplete adjustments for established risk factors and potential confounding variables (e.g. waist circumference or use of certain medications); as such, it was not possible to combine models in studies that adjusted for the same set of potential confounders. Third, although we used a random-effects model, some results of this meta-analysis should be interpreted cautiously (like all previously published meta-analyses $[7,8])$, given the relatively medium-to-high heterogeneity observed in the overall primary analysis $\left(I^{2}=60.7 \%\right.$, Figure 2$)$. However, as shown in the figure, this medium-to-high heterogeneity largely reflects differences in the methodologies used for the diagnosis of NAFLD (being this heterogeneity very low or absent for studies using blood biomarkers/scores, ICD codes or imaging techniques). In addition, the overall quality of eligible studies included in the meta-analysis was acceptable, suggesting a medium-to-low risk of bias according to the NOS scale. However, more detailed analyses of the causes of heterogeneity would require collaborative pooling of individual participant data from large cohort studies. Fourth, all the eligible studies used the MDRD or the CKD-EPI study equations to calculate eGFR (instead of direct GFR measurements to diagnose CKD), neither of which are reliable in severely obese individuals [44]. Finally, none of the eligible studies characterized NAFLD-associated kidney disease histologically. Therefore, it remains uncertain if NAFLD is associated with a specific type of kidney disease. However, it has been reported that NAFLD may promote kidney injury, mostly through atherothrombotic mechanisms [5] and, indeed, our meta-regression analyses showed a significant association between the proportion of patients with established hypertension at baseline and the risk of NAFLD-related CKD. Conversely, no significant effect of sex on the association between NAFLD and CKD risk was revealed by our meta-analysis. Probably, this results from failure of the eligible studies to 
adequately consider sex differences and sex hormones/menopausal status. In particular, none of these studies provided separate analyses by sex. Therefore, sex/age-specific analyses should be performed in future epidemiological studies, given that sex and menopausal status are acknowledged effect modifiers [45].

Notwithstanding these limitations, our study has important strengths. This meta-analysis incorporates data from large cohort studies from United States, Europe and Asia that are likely to be an accurate reflection of patients with NAFLD commonly seen in clinical practice. Moreover, we employed standardized risk estimates from all eligible studies to allow a consistent combination of estimates across studies. The large number of both patients with NAFLD at baseline and incident cases of CKD stage $\geq 3$ provided high statistical power to quantitatively assess the association between NAFLD and risk of incident CKD. Finally, although a selective reporting bias of studies cannot be definitely excluded, we also searched for "grey" literature and ruled out very low-quality studies. Thus, we believe that our comprehensive search has made it unlikely that any published studies were missed. In addition, the rank correlation Begg's test did not show any statistically significant asymmetry of the funnel plot, thus suggesting that publication bias was unlikely.

In conclusion, this largest and most updated meta-analysis to date shows that NAFLD is significantly associated with a $\sim 1.45$-fold increased risk of incident CKD stage $\geq 3$ over a median follow-up period of 9.7 years. However, additional research is needed to decipher the existing but complex link between the presence and severity of NAFLD and risk of incident CKD, and to further confirm whether improvement or resolution of NAFLD attenuates development and progression of CKD.

\section{ACKNOWLEDGEMENTS}

Authors Contributions: study concept and design: AM, GT; acquisition of data: AM, GP, GB, AC, GT; statistical analysis of data: AM; analysis and interpretation of data: AM, GT; drafting of 
the manuscript: GT; critical revision of the manuscript for important intellectual content: $A L$, JMS, HT and CDB.

Conflicts of Interest: JMS has acted as consultant for BMS, Boehringer Ingelheim, Echosens, Genfit, Gilead Sciences, Intercept Pharmaceuticals, Madrigal, Novartis, Pfizer, Roche and has received research funding from Gilead Sciences. All other authors have no potential conflicts of interest to disclose.

Sources of Funding: GT is supported in part by grants from the University School of Medicine of Verona, Verona, Italy. CDB is supported in part by the Southampton National Institute for Health Research (NIHR) Biomedical Research Centre.

Acknowledgments: We would like to thank Dr. Kristina Önnerhag (Skåne University Hospital Malmö, Sweden), who has kindly provided extra-information to us on her study, as reported in supplementary Table S3.

\section{REFERENCES}

1. Younossi ZM. Non-alcoholic fatty liver disease - A global public health perspective. J Hepatol 2019;70:531-544.

2. Byrne CD, Targher G. NAFLD: a multisystem disease. J Hepatol 2015;62:S47-S64.

3. Mantovani A, Scorletti E, Mosca A, Alisi A, Byrne CD, Targher G. Complications, morbidity and mortality of nonalcoholic fatty liver disease. Metabolism 2020;111S:154170.

4. Targher G, Chonchol MB, Byrne CD. CKD and nonalcoholic fatty liver disease. Am J Kidney Dis 2014;64:638-652.

5. Byrne CD, Targher G. NAFLD as a driver of chronic kidney disease. J Hepatol 2020;72:785-801.

6. Webster AC, Nagler EV, Morton RL, Masson P. Chronic kidney disease. Lancet 2017;389:1238-1252.

7. Musso G, Gambino R, Tabibian JH, Ekstedt M, Kechagias S, Hamaguchi M, et al. Association of non-alcoholic fatty liver disease with chronic kidney disease: a systematic review and meta-analysis. PLoS Med 2014;11:e1001680. 
8. Mantovani A, Zaza G, Byrne CD, Lonardo A, Zoppini G, Bonora E, et al. Nonalcoholic fatty liver disease increases risk of incident chronic kidney disease: a systematic review and meta-analysis. Metabolism 2018;79:64-76.

9. Stroup DF, Berlin JA, Morton SC, Olkin I, Williamson GD, Rennie D, et al. Meta-analysis of observational studies in epidemiology: a proposal for reporting. Meta-analysis Of Observational Studies in Epidemiology (MOOSE) group. JAMA 2000;283:2008-2012.

10. Higgins JPT, Green S (Eds). Cochrane Handbook for Systematic Reviews of Interventions Version 5.1.0 [updated March 2011]. The Cochrane Collaboration, 2011. Available from www.cochrane-handbook.org/Accessed 22 February 2017.

11. Higgins JP, Thompson SG. Quantifying heterogeneity in a meta-analysis. Stat Med 2002;21:1539-1518.

12. Egger M, Smith GD, Phillips AN. Meta-analysis: principles and procedures. BMJ 1997;315:1533-1537.

13. Tahan V, Canbakan B, Balci H, Dane F, Akin H, Can G, et al. Serum gammaglutamyltranspeptidase distinguishes non-alcoholic fatty liver disease at high risk. Hepatogastroenterology 2008;55:1433-1438.

14. Loomba R, Adams LA. Advances in non-invasive assessment of hepatic fibrosis. Gut 2020;69:1343-1352.

15. Ryu S, Chang Y, Kim DI, Kim WS, Suh BS. Gamma-glutamyltransferase as a predictor of chronic kidney disease in non-hypertensive and nondiabetic Korean men. Clin Chem 2007;53:71-77.

16. Chang Y, Ryu S, Sung E, Woo HY, Oh E, Cha K, et al. Nonalcoholic fatty liver disease predicts chronic kidney disease in non-hypertensive and nondiabetic Korean men. Metabolism 2008;57:569-576.

17. Targher G, Chonchol M, Bertolini L, Rodella S, Zenari L, Lippi G, et al. Increased risk of CKD among type 2 diabetics with nonalcoholic fatty liver disease. J Am Soc Nephrol 2008;19:1564-1570.

18. Arase $Y$, Suzuki F, Kobayashi M, Suzuki Y, Kawamura $Y$, Matsumoto $N$, et al. The development of chronic kidney disease in Japanese patients with non-alcoholic fatty liver disease. Intern Med 2011;50:1081-1087.

19. Targher G, Mantovani A, Pichiri I, Mingolla L, Cavalieri V, Mantovani W, et al. Nonalcoholic fatty liver disease is independently associated with an increased incidence of chronic kidney disease in patients with type 1 diabetes. Diabetes Care 2014;37:1729-1736.

20. Huh JH, Kim JY, Choi E, Kim JS, Chang Y, Sung KC. The fatty liver index as a predictor of incident chronic kidney disease in a 10-year prospective cohort study. PLoS One 2017;12:e0180951.

21. Shen ZW, Xing J, Wang QL, Faheem A, Ji X, Li J, et al. Association between serum Yglutamyltransferase and chronic kidney disease in urban Han Chinese: a prospective cohort study. Int Urol Nephrol 2017;49:303-312.

22. Kunutsor SK, Laukkanen JA. Gamma-glutamyltransferase and risk of chronic kidney disease: a prospective cohort study. Clin Chim Acta 2017;473:39-44.

23. Sinn DH, Kang D, Jang HR, Gu S, Cho SJ, Paik SW, et al. Nonalcoholic fatty liver disease and development of chronic kidney disease: a cohort study. J Hepatol 2017;67:12741280. 
24. Wilechansky RM, Pedley A, Massaro JM, Hoffmann U, Benjamin EJ, Long MT. Relations of liver fat with prevalent and incident chronic kidney disease in the Framingham Heart Study: A secondary analysis. Liver Int 2019;39:1535-1544.

25. Park H, Dawwas GK, Liu X, Nguyen MH. Nonalcoholic fatty liver disease increases risk of incident advanced chronic kidney disease: a propensity-matched cohort study. J Intern Med 2019;286:711-722.

26. Önnerhag $\mathrm{K}$, Hartman $\mathrm{H}$, Nilsson PM, Lindgren S. Non-invasive fibrosis scoring systems can predict future metabolic complications and overall mortality in non-alcoholic fatty liver disease (NAFLD). Scand J Gastroenterol 2019;54:328-334.

27. An JN, Joo SK, Koo BK, Kim JH, Oh S, Kim W. Portal inflammation predicts renal dysfunction in patients with nonalcoholic fatty liver disease. Hepatol Int 2020;14:798807.

28. Chon YE, Kim HJ, Choi YB, Hwang SG, Rim KS, Kim MN, et al. Decrease in waist-to-hip ratio reduced the development of chronic kidney disease in non-obese non-alcoholic fatty liver disease. Sci Rep 2020;10:8996.

29. Kaps L, Labenz C, Galle PR, Weinmann-Menke J, Kostev K, Schattenberg JM. Nonalcoholic fatty liver disease increases the risk of incident chronic kidney disease. United European Gastroenterol J 2020;8:942-948.

30. Lee DH, Jacobs DR Jr, Gross M, Steffes M. Serum gamma-glutamyltransferase was differently associated with microalbuminuria by status of hypertension or diabetes: the Coronary Artery Risk Development in Young Adults (CARDIA) Study. Clin Chem 2005;51:1185-1191.

31. El Azeem HA, Khalek el-SA, El-Akabawy H, Naeim H, Khalik HA, Alfifi AA. Association between nonalcoholic fatty liver disease and the incidence of cardiovascular and renal events. J Saudi Heart Assoc 2013;25:239-246.

32. Jang HR, Kang D, Sinn DH, Gu S, Cho SJ, Lee JE, et al. Nonalcoholic fatty liver disease accelerates kidney function decline in patients with chronic kidney disease: a cohort study. Sci Rep 2018;8:4718.

33. Chinnadurai R, Ritchie J, Green D, Kalra PA. Non-alcoholic fatty liver disease and clinical outcomes in chronic kidney disease. Nephrol Dial Transplant 2019;34:449-457.

34. Önnerhag K, Dreja K, Nilsson PM, Lindgren S. Increased mortality in non-alcoholic fatty liver disease with chronic kidney disease is explained by metabolic comorbidities. Clin Res Hepatol Gastroenterol 2019;43:542-550.

35. Taylor RS, Taylor RJ, Bayliss S, Hagström H, Nasr P, Schattenberg JM, et al. Association between fibrosis stage and outcomes of patients with nonalcoholic fatty liver disease: a systematic review and meta-analysis. Gastroenterology 2020;158:16111625.

36. Targher G, Byrne CD, Tilg H. NAFLD and increased risk of cardiovascular disease: clinical associations, pathophysiological mechanisms and pharmacological implications. Gut 2020;69:1691-1705.

37. Targher G, Byrne CD, Lonardo A, Zoppini G, Barbui C. Non-alcoholic fatty liver disease and risk of incident cardiovascular disease: a meta-analysis. J Hepatol 2016;65:589600.

38. Mantovani A, Dauriz M, Byrne CD, Lonardo A, Zoppini G, Bonora E, et al. Association between nonalcoholic fatty liver disease and colorectal tumours in asymptomatic 
adults undergoing screening colonoscopy: a systematic review and meta-analysis. Metabolism 2018;87:1-12.

39. Mantovani A, Petracca G, Beatrice G, Tilg H, Byrne CD, Targher G. Nonalcoholic fatty liver disease and risk of incident diabetes mellitus: an updated meta-analysis of 501,022 adult individuals. Gut 2020 Sep 16; gutjnl-2020-322572. doi: 10.1136/gutjnl2020-322572. Online ahead of print.

40. Ix JH, Sharma K. Mechanisms linking obesity, chronic kidney disease, and fatty liver disease: the roles of fetuin-A, adiponectin, and AMPK. J Am Soc Nephrol 2010;21:406412.

41. Targher G, Byrne CD. Non-alcoholic fatty liver disease: an emerging driving force in chronic kidney disease. Nat Rev Nephrol 2017;13:297-310.

42. Samuel VT, Shulman GI. Nonalcoholic fatty liver disease as a nexus of metabolic and hepatic diseases. Cell Metab 2018;27:22-41.

43. Meex RCR, Watt MJ. Hepatokines: linking nonalcoholic fatty liver disease and insulin resistance. Nat Rev Endocrinol 2017;13:509-520.

44. Levey AS, Inker LA. Assessment of glomerular filtration rate in health and disease: a state of the art review. Clin Pharmacol Ther 2017;102:405-419.

45. Lonardo A, Nascimbeni F, Ballestri S, Fairweather D, Win S, Than TA, et al. Sex differences in nonalcoholic fatty liver disease: state of the art and identification of research gaps. Hepatology 2019;70:1457-1469. 
Table 1. Subgroup analyses - Association between NAFLD and risk of incident CKD stage $\geq 3$, stratified by Newcastle-Ottawa Scale (NOS) category, length of study follow-up, degree of covariate adjustment, or study country.

\begin{tabular}{|c|c|c|c|c|c|}
\hline & $\begin{array}{l}\text { Hazard ratios } \\
(95 \% \mathrm{Cl})\end{array}$ & $\begin{array}{c}\text { Test for } \\
\text { overall effect }\end{array}$ & $\begin{array}{l}\text { Number } \\
\text { of studies }\end{array}$ & $\begin{array}{l}\text { Number of total } \\
\text { subjects included } \\
\text { (\% NAFLD; } n)\end{array}$ & $\begin{array}{l}\text { Heterogeneity } \\
\qquad I^{2}\end{array}$ \\
\hline NOS $\geq 8$ stars & 1.46 (95\% Cl 1.36-1.56) & $\begin{array}{l}Z=9.97 \\
p<0.0001\end{array}$ & 6 & $\begin{array}{c}1,186,267 \\
(27.8 \% ; 330,267)\end{array}$ & $67.1 \%$ \\
\hline NOS $<8$ stars & 1.34 (95\% Cl 1.16-1.54) & $\begin{array}{c}Z=4.04 \\
p<0.0001\end{array}$ & 4 & $\begin{array}{c}29,605 \\
(23.0 \% ; 6,821)\end{array}$ & $2.10 \%$ \\
\hline Follow-up $<5$ years & 1.38 (95\% Cl 1.05-1.81) & $\begin{array}{l}Z=2.31 \\
p=0.018\end{array}$ & 2 & $\begin{array}{c}49,759 \\
(33.6 \% ; 16,739)\end{array}$ & $73.1 \%$ \\
\hline Follow-up $\geq 5$ years & 1.46 (95\% Cl 1.37-1.57) & $\begin{array}{l}Z=10.98 \\
p<0.0001\end{array}$ & 8 & $\begin{array}{c}1,166,113 \\
(27.5 \% ; 320,349)\end{array}$ & $53.8 \%$ \\
\hline $\begin{array}{l}\text { Maximal covariate } \\
\text { adjustment* }\end{array}$ & 1.48 (95\% Cl 1.37-1.59) & $\begin{array}{c}Z=9.83 \\
p<0.0001\end{array}$ & 7 & $\begin{array}{c}1,204,286 \\
(27.8 \% ; 334,793)\end{array}$ & $64.0 \%$ \\
\hline $\begin{array}{l}\text { Minimal covariate } \\
\text { adjustment }\end{array}$ & 1.34 (95\% Cl 1.20-1.48) & $\begin{array}{c}Z=5.44 \\
p<0.0001\end{array}$ & 3 & $\begin{array}{c}11,586 \\
(19.8 \% ; 2,295)\end{array}$ & $0.0 \%$ \\
\hline Asia & 1.34 (95\% Cl 1.24-1.44) & $\begin{array}{l}Z=7.33 \\
p<0.0001\end{array}$ & 5 & $\begin{array}{c}82,475 \\
(29.6 \% ; 24,439)\end{array}$ & $0.0 \%$ \\
\hline Europe & 1.40 (95\% Cl 1.09-1.80) & $\begin{array}{l}Z=2.65 \\
p=0.007\end{array}$ & 3 & $\begin{array}{c}100,212 \\
(49.8 \% ; 49,899)\end{array}$ & $62.2 \%$ \\
\hline United States & 1.58 (95\% Cl 1.51-1.65) & $\begin{array}{l}Z=20.35 \\
p<0.0001\end{array}$ & 2 & $\begin{array}{c}1,033,185 \\
(25.4 \% ; 262,750)\end{array}$ & $0.0 \%$ \\
\hline
\end{tabular}

$N B$ : In these subgroup analyses, we have analyzed all the eligible studies that were included in the Figure 2 ( $\mathrm{n}=10$ studies).

*Maximal adjustment includes only studies that have adjusted their results for at least the following covariates: age, sex, body mass index, smoking history, pre-existing diabetes (or plasma glucose levels), hypertension, dyslipidemia (or plasma lipid levels), and baseline eGFR. 


\section{FIGURE LEGENDS}

Figure 1. The PRISMA flow diagram for search and selection processes of the meta-analysis.

Figure 2. Forest plot and pooled estimates of the effect of NAFLD on the risk of incident CKD stage $\geq 3$ in 10 eligible studies, stratified by the methodology used for the diagnosis of NAFLD.

Supplementary Figure S1. Univariable meta-regression analyses. A meta-analysis of the association of age (panel A), body mass index (panel B), female sex (panel C), proportion of hypertension (panel D) or pre-existing diabetes (panel E) with the risk of incident CKD stage $\geq 3$ (for the 10 eligible studies included in Figure 2).

Supplementary Figure S2. Meta-analysis estimates, given named study is omitted (for the 10 eligible studies included in Figure 2). The effect size was expressed as random-effects HRs and $95 \% \mathrm{Cls}$ for all studies.

Supplementary Figure S3. Funnel plot of standard error by log-hazard ratio for the risk of incident CKD stage $\geq 3$ (for the 10 eligible studies included in Figure 2). $P$-values by the rank correlation Begg's test. 